\title{
The use of atypical antipsychotics in dementia: rethinking Simpson's paradox
}

\section{Introduction}

Higher rates of cerebrovascular adverse events (CVEs) and mortality for dementia patients taking active drugs were reported during industrysponsored clinical trials of atypical antipsychotics for the treatment of behavioral and psychological symptoms of dementia (BPSD). Since then, the use of antipsychotics for BPSD has not been recommended on the basis of advisory warnings issued by regulatory authorities in several countries. Nevertheless, there are currently no demonstrated pharmacologic alternatives. Although early published and unpublished data indicated a risk, few subsequent publications have supported the initial finding. In order to update earlier comments published in International Psychogeriatrics (Shah and Suh, 2005), this editorial briefly introduces subsequent reports, summarizes the discrepant findings of experimental and non-experimental studies, highlights the effect of "Simpson's paradox", which causes us to throw doubt on conclusions based on meta-analyses or pooled-analyses, explains this discrepancy in the light of current knowledge, and concludes with practical recommendations regarding the use of atypical antipsychotics in dementia.

\section{Reports from experimental randomized clinical trials (RCTs)}

In 2003, Brodaty et al. (2003) reported higher rates of CVEs and mortality in patients with dementiaassociated agitation and psychosis who were treated with risperidone compared with those receiving placebo in a randomized trial. In 2004, the U.K. Committee of Safety of Medicines (CSM) informed clinicians that risperidone and olanzapine should not be used to treat BPSD because of increased risk of strokes with both drugs and an increased risk of mortality with olanzapine. The CSM cited the following evidence: (i) a metaanalysis of randomized placebo-controlled studies of risperidone in dementia demonstrated a threefold increase in strokes and; (ii) a pooledanalysis of randomized placebo-controlled studies of olanzapine in dementia demonstrated a three-

First published online 26 February 2009. fold increase in strokes and a two-fold increase in mortality (CSM, 2004). In 2005, the U.S. Food and Drug Administration (FDA) issued a public health advisory warning alerting health care providers and the public about new safety information regarding all atypical antipsychotic medications. When 17 placebo-controlled trials relating to the use of olanzapine, aripiprazole, risperidone and quetiapine in dementia patients with BPSD $(\mathrm{N}=5106)$ were pooled, the mortality of the drug-treated group was 1.6 or 1.7 times that of the placebo group $(4.5 \%$ vs $2.6 \%$ ), predominantly due to heart-related or infectious causes (FDA, 2005).

\section{Reversal of results when the groups are combined: Simpson's paradox}

Simpson's paradox is a statistical paradox wherein the successes of groups seem reversed when the groups are combined (Wagner, 1982). Simpson's paradox occurs when we combine data. In Figure 1, the overall mortality rate of Treatment $\mathrm{A}$ is $22 \%$ (77/350), while that of Treatment $\mathrm{B}$ is $17 \%$ (61/350). But we should not simply conclude that the mortality rate of Treatment $\mathrm{A}$ is higher than that of Treatment B. It is because Treatment A is mostly used for patients with high risk factors ( $\mathrm{Mo}_{\mathrm{Tx} \mathrm{A}}$ : Mo $\mathrm{Tx}_{\mathrm{B}}=27 \%: 31 \%$ ) while Treatment $\mathrm{B}$ is mostly used for patients without such high risk factors (Mo Tx A : $\left.\mathrm{Mo}_{\mathrm{Tx} \mathrm{B}}=7 \%: 13 \%\right)$. The successes of Treatment $\mathrm{A}$ are reversed when the two groups are combined.

A meta-analysis combines results from several randomized controlled trials (RCTs) of similar design in order to obtain a larger number of patients to improve the evaluation of whether statistically reliable differences exist between comparison groups. This systematic review tends to overcome the subjective and often biased traditional narrative reviews which most often do not specify the source of information and fail to perform a standardized assessment of the methodological quality of studies (Teagarden, 1989). However, meta-analyses are by no means perfect. Some metaanalyses of small trials were later contradicted by a single large randomized trial on the same topic. Well-known examples are the effects of nitrates (Yusuf et al., 1988; GISSI, 1994) and magnesium 


\begin{tabular}{lcl}
\hline Mortality & $\begin{array}{c}\text { TreatMent A } \\
\text { 22\% (77/350) }\end{array}$ & $\begin{array}{l}\text { TREATMENT B } \\
17 \%(61 / 350)\end{array}$ \\
\hline & & \\
& & \\
\hline MorTALiTY & & \\
\hline Without high risk factor & $7 \%(6 / 87)$ & $13 \%(36 / 270)$ \\
With high risk factor & $27 \%(71 / 263)$ & $31 \%(25 / 80)$ \\
Both & $22 \%(77 / 350)$ & $17 \%(61 / 350)$ \\
\hline
\end{tabular}

Figure 1. Simpson's paradox, a statistical paradox wherein the successes of groups seem reversed when the groups are combined.

(Teo et al., 1991; ISIS-4, 1995) on mortality in acute myocardial infarction, and the effect of aspirin on the risk of pre-eclampsia (Imperiale and Petrullis, 1991; CLASP Collaborative Group, 1995). Obviously the quality of component trials is of crucial importance: if the raw material is flawed, the resulting meta-analysis will also be flawed ("garbage in, garbage out") (Verstraete, 2002).

The aforementioned regulatory agencies stated that they conducted meta-analyses or pooledanalyses using simple methodology to calculate risks of mortality or CVE based on combined tables summing up results across different studies (CSM, 2004; FDA, 2005). The conclusions made by the regulatory agencies from the meta- and pooled-analyses are subject to the same potential for bias, the so-called Simpson's paradox ("the conclusion is reversed"). If a very large trial is poorly conducted and is part of a meta-analysis, the results of the meta-analysis can be adversely impacted by that trial. Data were combined by agent (i.e. risperidone only, olanzapine only) (CSM, 2004; Haupt et al., 2006) or even by the supposed same category (i.e. atypical antipsychotics including quetiapine, ziprasidone, aripiprazole, risperidone and olanzapine) (FDA, 2005; Schneider et al., 2006a). There is no rationale for considering all atypical antipsychotics to be the same. Profiles of adverse events vary widely across different atypical antipsychotics. Even sociodemographic and clinical characteristics (i.e. age, gender, levels of cognition and behavioral disturbance) of the active treatment group and the comparator group vary widely across RCTs. The meta-analyses seem to assume that all atypical antipsychotics have the same efficacy and safety. In addition, none of the RCTs were initially designed to look for CVEs or mortality.

\section{Reports from recent non-experimental studies}

Even though a very large RCT is the most reliable way of obtaining reproducible results, future trials of antipsychotics for BPSD are unlikely. We need to scrutinize the current published literature. There have been reports from non-experimental studies which contradict those of the U.K.'s CSM and the U.S.A.'s FDA.

In 2005, Suh and Shah reported results of a one-year prospective study comparing antipsychotic users and antipsychotic non-users in a nursing home $(\mathrm{N}=273)$. We reported that the mortality rate in those who had not received antipsychotics $(26.8 \%)$ was higher than that in those who had received antipsychotics $(20.8 \%)$. The mortality rates of the two groups were statistically significantly different, even after controlling for possible confounding factors (Suh and Shah, 2005).

In 2006, Schneider and his colleagues reported results of a prospective study entitled Clinical Antipsychotic Trials of Intervention EffectivenessAlzheimer's Disease (CATIE-AD), which was supported by the U.S. National Institute of Mental Health (NIMH) (Schneider et al., 2006b). In this study, doses were adjusted as needed, and 421 outpatients were followed for up to 36 weeks. Neither the incidence of CVE (two cases in the olanzapine group, one in the quetiapine group, one in the risperidone group, one in the placebo group; $\mathrm{p}=0.92$ ) nor mortality rates (one case in the olanzapine group, three in the quetiapine group, one in the risperidone group, three in the placebo group; $\mathrm{p}=0.68$ ) were statistically different in the overall comparison. The CATIE-AD study reported that the sum total of the risk/benefit equation of atypical antipsychotic therapy was no greater than that achieved by placebo.

In 2006, Nonino and his colleagues reported that the long-term survival of 294 dementia patients with BPSD treated with atypical antipsychotics may not significantly differ from that of dementia patients not treated with these drugs, using data from a population-based cohort study conducted in Modena, Italy (Nonino et al., 2006).

In 2006 and 2007, Barnett and his colleagues reported a retrospective review of the clinical 
outcomes of a large cohort of elderly patients admitted to hospital for pneumonia or for dementia. They found no increased risk of mortality in pneumonia patients treated with atypical antipsychotics, and no increased risk for CVE-related hospital admission in dementia patients treated with atypical antipsychotics (Barnett et al., 2006; 2007).

In 2007, Raivio and her colleagues reported results of a two-year prospective study with 254 very frail dementia patients from seven nursing homes and two hospitals in Finland, which concluded that neither atypical nor conventional antipsychotics increases mortality or hospital admissions among dementia patients (Raivio et al., 2007).

A population-based retrospective cohort study (1997-2002) in Canada that included 11,400 patients found no difference in the rate of strokes requiring hospitalization among users of the conventional antipsychotics risperidone and olanzapine (Herrmann et al., 2004). The same research group reported an increased risk of death in the use of atypical antipsychotics compared to nonuse among dementia patients, using data including the additional year of 2003 (1997-2003) (Gill et al., 2007).

In summary, none of the reports except one (Gill et al., 2007) support the reports from previous experimental clinical trials. Almost all nonexperimental clinical studies have shown no increase in the risk of CVE and mortality associated with use of atypical antipsychotics and the results also suggest that they may be associated with a lower mortality rate than conventional antipsychotics (Nasrallah et al., 2004; Schneeweiss et al., 2007; Trifiro et al., 2007).

\section{Older people in the experimental studies are at higher risk of harm}

Some may argue that evidence from experimental RCTs should be preferred when reports are contradictory. However, in terms of the incidence of CVE and mortality, older people in the experimental studies are at higher risk of harm than older people in the non-experimental studies. An RCT is a type of scientific experiment most commonly used in testing a trial medication versus a placebo or the standard medical alternative as an active comparator, usually to assess the safety and efficacy of a trial medication on a specific kind of patient group. The study design can provide the most compelling evidence that the trial medication causes the expected effect on human health. Therefore, RCTs are considered the most reliable form of scientific evidence in healthcare because they eliminate spurious causality and bias. As the name suggests, RCTs involve the random allocation of different treatments to patients. This ensures that known and unknown confounding factors are evenly distributed between groups. However, patients who are allocated to the active treatment group may be at higher risk of serious adverse events than those allocated to the placebo group. This might be due to the following reasons:

(i) Contrary to steadily declining cognition and function in dementia patients, BPSD is not constantly presenting, but sometimes spontaneously goes into remission. BPSD often fluctuates and can have an episodic course. Most BPSD are expected to respond to antipsychotics within one to two weeks, and most symptoms go into remission soon. Then, in usual clinical practice, clinicians maintain antipsychotics for a time-limited period and then taper and discontinue the medication. In clinical practice, medication for BPSD is often used on an "as needed" basis. However, in RCTs, patients in the active treatment group continue to take antipsychotics to the end of the trial, most for 8 to 36 weeks, even though they do not need antipsychotics any longer because their symptoms are now very mild or in remission. This means that patients in the active treatment group are unnecessarily exposed to a higher risk of harm.

(ii) The experimental condition itself may put patients on the active treatment at higher risk of harm. Once recruited for RCTs, every patient takes the trial medication as scheduled. To guarantee the safety of patients, study protocols of RCTs of antipsychotics for BPSD did not exclude patients using drugs which had been regularly used to control stable general medical conditions (e.g. hypertension, diabetes) throughout the trial period. Older people are dangerously overmedicated in the real world, yet many frequently omit to take medications. However, in the experimental condition, they have to take medications without omission throughout the trial period. The compulsory continuous use of these different kinds of drugs in RCTs may increase the chance of drug-disease interactions or drugdrug interactions with the trial medication. In RCTs of antipsychotics for BPSD, by fixing doses and requiring that patients be kept on medication throughout the trial, many patients are placed at increased risk for adverse events and do not have the potential to benefit from individualized dosing adjustments. By comparison, in usual clinical practice, medication might have been discontinued, decreased, increased or switched. Thus, the clinical trials may reflect both more adverse events and less efficacy than are actually experienced by patients in "real life". 


\section{Antipsychotics: chemical cosh or sovereign remedy?}

Antipsychotics, the primary treatment agent for schizophrenia and other psychoses, have long been suspected to increase the risk of serious ventricular arrhythmias and sudden cardiac death. An increased risk of sudden cardiac death is consistent with the dose-related effects of antipsychotics on cardiac electrophysiologic properties. Haloperidol and sertindole block repolarizing potassium currents in vitro, hypothesized to be the mechanism underlying drug-induced torsades de pointes (Ray et al., 2001). All drugs that cause torsades de pointes prolong the QTc interval and blind the potassium rectifier channel. Although pimozide, sertindole, droperidol and haloperidol have been documented to cause torsades de pointes and sudden death, the most marked risk is with thioridazine. Except for haloperidol, these drugs are not widely used today, or have disappeared from the market. It is believed that none of the currently available atypical antipsychotics may cause torsades de pointes and sudden death within their therapeutic range.

In usual clinical practice, misuse of insulin, oral hypoglycemic agents, digoxin, warfarin, tricyclic antidepressants and sedative-hypnotics can be fatal. Balancing these known risks with the positive effects of treatment poses a challenge. All currently available antipsychotics have the potential to cause serious adverse events. Like other medications, antipsychotics are also therapeutic agents that can cause both benefit and harm, like a double-edged sword that should be used judiciously. "Chemical cosh" and "sovereign remedy" are names for the two different faces of the same agent. Causes of BPSD in dementia patients are heterogeneous and complex to manage. All clinicians should clearly recognize that atypical antipsychotics are not the treatment of choice for BPSD. Antipsychotics should not be viewed as broad-spectrum agents. The efficacy and safety of antipsychotics should be more narrowly defined and considered. Given that future trials are unlikely and antipsychotics do not generally have approval as agents to treat BPSD, it will be difficult to explore the underlying mechanism systematically. Therefore, we should make greater efforts to find clues within the existing data and literature.

\section{Possible mechanisms leading to CVE and death after use of antipsychotics}

The biological mechanism leading to CVE and death after use of atypical antipsychotics for BPSD is obscure. Possible biological mechanisms to account for the association between CVE and atypical antipsychotics might include thromboembolic effects, cardiovascular effects (such as orthostatic hypotension and arrhythmias), hyperprolactinemia leading to atherosclerosis, and excessive sedation, resulting in dehydration and hemoconcentration (Herrmann et al., 2005). In the FDA's analysis, heart-related events (heart failure and sudden death) and infections (mostly pneumonia) accounted for many deaths. First, this may be due to a direct medication effect of antipsychotics. The anticholinergic effect of antipsychotics affects blood pressure, heart rate and QTc interval, causing conduction delays which may lead to ventricular tarchycardia or torsades de pointes. Second, possible drug-drug interactions should be considered. Sedation is associated with antipsychotics in clinical practice. Concomitant use of benzodiazepines can cause deep sedation in dementia patients also receiving antipsychotics, which could exacerbate pre-existing cognitive impairment and increase the risk of aspiration pneumonia. On the other hand, in a meta-analysis of risperidone, the mortality rate in furosemide-treated dementia patients was $7.3 \%$ for the risperidone group versus $4.1 \%$ for the placebo group (Haupt et al., 2006). A recent Spanish report from a longitudinal observational study indicates that neither the presence of cardiac disease nor the metabolic syndrome explained the increased mortality rate in dementia patients who were treated with risperidone or olanzapine (Vilalta-Franch et al., 2008). Third, it may be the pathophysiology that prompted the use of antipsychotics (drugdisease interaction: use of antipsychotics to treat visual hallucination in patients with dementia with Lewy bodies). It is supposed that dementia patients with dominant synucleinopathy (i.e. dementia with Lewy bodies) are more vulnerable and sensitive to antipsychotics, compared to dementia patients with dominant amyloidopathy (i.e. Alzheimer's dementia).

The statistically significant increased risk of harm reported from RCTs looks marginal. Herrmann and colleagues wrote that assuming the relative risk of stroke was 1.4, this would translate into two extra strokes per 1,000 person-years of treatment (Herrmann et al., 2005). This strongly suggests that some associated factors are involved: (a) drug-drug interaction (i.e. co-administered benzodiazepine or frusemide); (b) drug-disease interaction (i.e. neuroleptic sensitivity in patients with Lewy body disease); (c) direct medication effect (i.e. torsades de pointes); or (d) findings due to incidental uneven distribution of known and unknown confounding factors in spite of random allocation of patients. 


\section{Conclusion}

Atypical antipsychotics for the treatment of BPSD are modestly effective when used judiciously, and there are as yet no demonstrated, effective pharmacologic alternatives. However, it would not be prudent to prescribe other medications in lieu of antipsychotics in the belief that they are as effective as or safer than atypical antipsychotics. There is no evidence either for efficacy or for adverse events for drugs other than antipsychotics. In the face of even more limited data for alternative pharmacotherapy, the use of atypical antipsychotics for the treatment of BPSD should not be suspended. The use of atypical antipsychotics for BPSD is not contra-indicated. Atypical antipsychotics will continue to be prescribed for BPSD in the absence of more effective, better tolerated and safer alternatives. A decision to use antipsychotics to treat fragile dementia patients must be made on the basis of individual circumstances. Clinicians may first consider nonpharmacologic interventions. Use of atypical antipsychotics would follow failed attempts at treating BPSD with non-pharmacological interventions and then with medications other than atypical antipsychotics. When using atypical antipsychotics, it is important to treat for a time-limited period and then taper and discontinue. The administration on a "prescribedrequired-as-needed" (PRN) basis might be better.

\section{Conflict of interest}

None.

\section{Acknowledgment}

Portions of this article were presented at the 10th Springfield Symposium, Hong Kong, 29 February 2008.

\section{GuK-HeE SuH}

Department of Psychiatry, Hallym University Medical Center, Hangang Sacred Heart Hospital,

Yeongdeungpo-Dong, Seoul, Korea

Email: suhgh@chol.com

\section{References}

Barnett, M. J., Perry, P. J., Alexander, B. and Kaboli, P. J. (2006). Risk of mortality associated with antipsychotic and other neuropsychiatric drugs in pneumonia patients. fournal of Clinical Psychopharmacology, 26, 182-187.

Barnett, M. J., Wehring, H. and Perry, P. J. (2007). Comparison of risk of cerebrovascular events in an elderly VA population with dementia between antipsychotic and nonantipsychotic users. Fournal of Clinical Psychopharmacology, 27, 595-601.

Brodaty, H. et al. (2003). A randomized placebo-controlled trial of risperidone for the treatment of aggression, agitation, and psychosis of dementia. Fournal of Clinical Psychiatry, 64, 134-143.

CLASP Collaborative Group (1994). CLASP: a randomized trial of low-dose aspirin for the prevention and treatment of pre-eclampsia among 9364 pregnant women. Lancet, 343, 619-629.

Committee of Safety of Medicines (CSM) (2004). Atypical antipsychotics and stroke. http://medicines.mhra.gov.uk/ ourwork/monitorsafequalmed/messages/risperidoneclinicaltrialdata final.pdf. Last accessed 13 May 2004.

Food and Drug Administration (FDA) (2005). Public health advisory: deaths with antipsychotics in elderly patients with behavioral disturbances. http://www.fda.gov/cder/drug/advisory/antipsychotics.htm. Last accessed 22 April 2005.

Gill, S. S. et al. (2007). Antipsychotics use and mortality in older adults with dementia. Annals of Internal Medicine, 146, 775-786.

Gruppo Italiano per lo Studio della Streptochinasi Nell'Infarco Miocardico (GISSI) (1994). GISSI-3: effects of lisinopril and transdermal glyceryl trinitrate singly and together on 6-week mortality and ventricular function after acute myocardial infarction. Lancet, 343, 1115-1122.

Haupt, M., Cruz-Jentoft, A. and Jeste, D. (2006). Mortality in elderly dementia patients treated with risperidone. Fournal of Clinical Psychopharmacology, 26, 566-570.

Herrmann, N., Mamdani, M. and Lanctôt, K. L. (2004). Atypical antipsychotics and risk of cerebrovascular accidents. American fournal of Psychiatry, 161, 1113-1115.

Herrmann, N., Lanctôt, K. and Mandani, M. (2005). Psychotropic medication and stroke outcome. American Fournal of Psychiatry, 162, 1027-1028.

Imperiale, T. E. and Petrullis, A. S. (1991). A meta-analysis of low-dose aspirin for the prevention of pregnancy-induced hypertensive disease. $\mathcal{F} A M A, 266$, 261-265.

ISIS-4 Collaboration Group (1995). ISIS-4: a randomized factorial trial assessing early oral captopril, oral mononitrate, and intravenous magnesium in 58,050 patients with suspected acute myocardial infarction. Lancet, 345, 669-685.

Nasrallah, H. A., Whit, T. and Nasrallah, A. (2004). Lower morality in geriatric patients receiving risperidone and olanzapine versus haloperidol. American fournal of Geriatric Psychiatry, 12, 437-439.

Nonino, F., Girolamo, G., Gamberini, L. and Goldoni, C. A. (2006). Survival among eldrly Italian patients with dementia treated with atypical antipsychotics: observational study. Neurological Sciences, 27, 375-380.

Raivio, M. M., Laurila, J. V., Strandberg, T. E., Tilvis, R. S. and Pitkala, K. H. (2007). Neither atypical nor conventional antipsychotics increase mortality or hospital admissions among elderly patients with dementia: a two-year prospective study. American fournal of Geriatric Psychiatry, 15, 416-424.

Ray, W. A., Meredith, S., Thapa, P. B., Meador, K. G., Hall, K. and Murray, K. T. (2001). Antipsychotics and 
the risk of sudden cardiac death. Archives of General Psychiatry, 58, 1161-1167.

Schneeweiss, S., Setoguch, S., Brookhart, A., Dormuth, C. and Wang, P. S. (2007). Risk of death associated with the use of conventional versus atypical antipsychotics among elderly patients. Canadian Medical Association fournal, 176, 627-632.

Schneider, L. S., Dagerman, M. S. and Insel, P. S. (2006a). Efficacy and adverse effects of atypical antipsychotics for dementia: meta-analysis of randomized, placebo-controlled trials. American fournal of Geriatric Psychiatry, 14, 191-210.

Schneider, L. S. et al. (2006b). Effectiveness of atypical antipsychotics in patients with Alzheimer's disease. New England fournal of Medicine, 355, 1525-1538.

Shah, A. and Suh, G. H. (2005). A case for judicious use of risperidone and olanzapine in behavioral and psychological symptoms of dementia (BPSD). International Psychogeriatrics, 17, 12-22.

Suh, G. H. and Shah, A. (2005). Effect of antipsychotics on mortality in elderly patients with dementia: a 1-year prospective study in a nursing home. International Psychogeriatrics, 17, 429-441.
Teagarden, J. R. (1989). Meta-analysis: whither narrative review? Pharmacotherapy, 9, 274-281.

Teo, K. K. et al. (1991). Effects of intravenous magnesium in suspected acute myocardial infarction: overview of randomized trials. British Medical fournal, 303, 14991503.

Trifiro, G. et al. (2007). All-cause mortality associated with atypical and typical antipsychotics in demented outpatients. Pharmacoepidemiology and Drug Safety, 16, 538-544.

Verstraete, M. (2002). Value and limitation of meta-analysis. Pathophysiology of Haemostasis and Thrombosis, 32, 278281.

Vilalta-Franch, J., Lopez-Pousa, S., Garre-Olmo, J., Turon-Estrada, A. and Pericot-Nierga, I. (2008). Mortality rates in patients with Alzheimer's disease treated with atypical neuroleptic drugs. Revista de Neurologia, 46, 129-134.

Wagner, C. H. (1982). Simpson's paradox in real life. American Statistician, 36, 46-48.

Yusuf, S., Collins, R., MacMahon, S. and Peto, R. (1988). Effect of intravenous nitrates on mortality in acute myocardial infarction: an overview of the randomized trials. Lancet, 14, 1088-1092. 